\title{
Adaptando los ODS a lo Local mediante la Educación para el Desarrollo. La Experiencia de la Estrategia de la Ciudad de Valencia
}

\author{
Localizing SDGs through Development Education. The \\ Experience of the Valencian City Strategy
}

\author{
Alejandra Boni ${ }^{1}$ * \\ Sergio Belda-Miquel 1,2 \\ Carola Calabuig-Tormo ${ }^{1}$ \\ María Alejandra Millán-Franco ${ }^{1}$ \\ Alba Talón-Villacañas ${ }^{1}$ \\ ${ }^{1}$ Universitat Politècnica de València, España \\ ${ }^{2}$ Universitat de València, España
}

\begin{abstract}
Este artículo presenta la propuesta de Educación para el Desarrollo que se recoge en la Estrategia para la Educación no formal de la ciudad de Valencia y su relación con los Objetivos de Desarrollo Sostenible (ODS). El contenido de la Estrategia se basa en tres ejes fundamentales: 1) un enfoque territorial en el barrio o distrito; 2) la importancia de la coordinación entre actores municipales y las ONGD para desarrollar las propuestas educativas y 3 ) una serie de temáticas y metodologías prioritarias. La correspondencia de la Estrategia con los oDs ha sido interpretada empleando un marco de análisis pensado para la innovación transformadora. Esta interpretación distingue entre oDs que definen los sistemas sociotécnicos o áreas de aplicación, los que definen la direccionalidad y los que indican las condiciones de contexto. Esta interpretación puede contribuir a aumentar la reflexión sobre los objetivos de transformación de las acciones educativas de una manera sinérgica, evitando perspectivas compartimentadas. Por otro lado, pone de manifiesto la importancia de las redes y coordinación entre actores y la ciudad y sus barrios. Estos son los espacios que necesitan ser transformados y a partir de los cuales pueden partir las transformaciones.
\end{abstract}

Descriptores: Educación para el desarrollo; Educación para la ciudadanía global; Objetivos de desarrollo sostenible; Estrategia; Política pública.

\begin{abstract}
This article presents the relationship between the Strategy of Non-Formal Development Education of the city of Valencia and the Sustainable Development Goals (SDG). The content of the Strategy is based on three fundamental axes: 1) a territorial approach in the neighbourhood or district; 2) the importance of coordination between municipal actors and NGDOS to develop educational proposals and 3) a series of priority themes and methodologies. The correspondence of the Strategy with the SDGS has been interpreted using an analytical framework designed for transformative innovation. This interpretation distinguishes between SDGS that define socio-technical systems or areas of application; those that define directionality and those that indicate context conditions. This interpretation can contribute to increase the reflection on the transformatory potential of educational actions in a synergic way, avoiding compartmentalized perspectives. On the other hand, it highlights the importance of networks and coordination between actors in the city and its neighbourhoods. These are the spaces that need to be transformed and from which transformations can start.
\end{abstract}

Keywords: Development education; Global citizenship education; Sustanaible development goals; Strategy; Public policy.

*Contacto: aboni@dpi.upv.es

ISSN: 2254-3139

www.rinace.net/riejs/

revistas.uam.es/riejs
Recibido: $\quad 11$ de febrero de 2019

$1^{\text {a }}$ Evaluación: 22 de abril de 2019

$2^{\text {a }}$ Evaluación: 5 de mayo de 2019

Aceptado: $\quad 9$ de mayo de 2019 


\section{Introducción}

Aunque la Agenda 2030 para el Desarrollo Sostenible y sus Objetivos de Desarrollo Sostenible (ODS), aprobados por la Asamblea General de Naciones Unidas en 2015, coloquen a los Estados como principales interlocutores para su implementación, las entidades locales no son ajenas a su puesta en práctica. En última instancia, serán los municipios los espacios en los que muchas de las propuestas de la Agenda 2030 se materialicen. Asimismo, en el caso valenciano, la Agenda 2030 está especialmente presente en el nivel autonómico tal y como se desprende de las distintas normativas promulgadas recientemente (IV Plan Director y Ley de Cooperación y Desarrollo Sostenible, ambas de 2017).

En este marco, el rol que puede jugar la Educación para el Desarrollo (en adelante ED) es múltiple y excede el cumplimiento del oDs 4, que se refiere a una educación de calidad, y su meta 4.7, que específicamente se refiere a:

De aquí a 2030, asegurar que todos los alumnos adquieran los conocimientos teóricos
y prácticos necesarios para promover el desarrollo sostenible, entre otras cosas
mediante la educación para el desarrollo sostenible y la adopción de estilos de vida
sostenibles, los derechos humanos, la igualdad de género, la promoción de una cultura
de paz y no violencia, la ciudadanía mundialy la valoración de la diversidad cultural
y de la contribución de la cultura al desarrollo sostenible. (Asamblea General de
NNUU, 2015, p. 20)

Además de contribuir al cumplimiento de este ODS, la propuesta de educación que contiene la Estrategia de ED de la ciudad de Valencia (en adelante la Estrategia) puede ser vista como un medio que contribuya a la Agenda 2030 en su globalidad. Y, más concretamente, a la adquisición de numerosos oDs relacionados con el cambio climático, las desigualdades, el género, la pobreza, la creación de redes locales y globales, la paz y la vida en unas ciudades más inclusivas, equitativas y sostenibles.

En la sección siguiente de este artículo ilustraremos las características principales de la Estrategia para, a continuación, discutir su relación con los ODS. Para esto último, emplearemos una interpretación de los ODS y los procesos de innovación transformadora desarrollada por Schot y otros (2018) donde el acento se coloca en la transformación y los ODS son vistos como ámbitos, condiciones y objetivos de la transformación. De esta manera, podemos entender la contribución de la ED de una forma mucho más amplia, multisectorial y con un mayor alcance.

\section{La estrategia de ED de la ciudad de Valencia}

\subsection{La perspectiva transformadora de la ED que guía la estrategia}

Desde el comienzo del proceso que llevaría a la elaboración de la Estrategia, se acordó que la perspectiva más adecuada de la ED que podría guiarlo es la que la entiende como educación para la ciudadanía global. Como ya se ha destacado por la literatura (Boni, 2011; Boni y León, 2013; Mesa, 2000) la ED ha caminado desde una visión asistencialista y eurocéntrica hacia un enfoque interdependiente y global. La llamada quinta generación de la ED la entiende como:

Proceso de aprendizaje social compartido y como espacio de confluencia de actores orientados a la transformación social. Una ED dirigida a la construcción de ciudadanía global mediante la participación y la incidencia política exige la 
articulación de actores y procesos educativos, sociales y culturales en espacios de sinergia y cooperación. (CONGDE, 2011, p. 4)

A pesar de la diversidad de definiciones del término que nos atañe (por ejemplo, ver Boni et al., 2016; INCYDE, 2016), la anterior definición nos sirve para destacar los ejes clave que guían la concepción de ED de la Estrategia:

ED como un proceso de aprendizaje social. La sociedad del siglo XXI necesita ser consciente de la importancia de alargar la etapa de aprendizaje más allá de la edad temprana, tal como apunta el nuevo consenso sobre desarrollo de la Comisión Europea (2017). Además, este proceso de aprendizaje debe adquirir un carácter colectivo y transformador, a semejanza de la Educación Popular en la que se inspira. Así, el carácter pedagógico transformador de la ED consiste en entender la complejidad de la realidad social y su dinamismo, propiciar en el sujeto el deseo de querer conocer y experimentar en relación con las demás personas, y movilizar para actuar sobre el propio entorno.

En segundo lugar, la construcción de una ciudadanía global a la que se refiere la definición de la CONGDE dirige este continuo aprender hacia una determinada forma de entender y actuar en el mundo social. Esta forma de comprender y actuar implica posicionarse frente a la globalización y sus consecuencias desde la comprensión holística de los problemas sociales que obstaculizan el desarrollo de capacidades de las personas (HEGOA, 2014). La futura ciudadanía global será aquella que piensa globalmente y actúa localmente, que conoce la relación que existe entre su entorno local con el todo global, que se sienta parte de los problemas mundiales y de las soluciones (Ortega-Carpio, Cordón y Sianes, 2013). Así, esta concepción de la ED implica: a) promover valores y actitudes clave de una ciudadanía mundial responsable; b) permitir un debate público e informado sobre las cuestiones que afectan al desarrollo y c) apoyar y dar legitimidad a las ONGD y a los gobiernos para que actúen decididamente a favor de la justicia global (Boni et al., 2016).

En tercer lugar, el carácter movilizador de la ED incide en la participación y en la incidencia política, ejes primordiales para fomentar un sujeto consciente y activo en su entorno ya que, como recoge la CONGDE:

\begin{abstract}
Mediante la ED se analiza el modelo de desarrollo actual, se examinan las causas de los problemas y sus consecuencias entendiéndolas como globales, planteando alternativas y contribuyendo a generar una ciudadanía crítica, activa y solidaria, implicada en producir los cambios necesarios en el modelo de desarrollo vigente y en las políticas que obstaculizan el desarrollo, para lograr un planeta equitativo y justo globalmente. Todo esto sin perder la mirada a lo local. (CONGDE, 2014, p. 6)
\end{abstract}

En cuarto y último lugar, la definición escogida expone que el proceso de la ED conlleva la creación de espacios de sinergia y cooperación. Vivir en la sociedad global hace necesario aumentar los canales de comunicación para el desarrollo. Tejer redes locales y facilitar espacios públicos de encuentro y debate debe así formar parte del proceso de educar para el desarrollo en procesos no formales.

En resumen, la visión de la ED que guía la Estrategia entiende que esta ha de tener como objetivo:

La promoción de una ciudadanía global que pueda ejercer derechos y responsabilidades para la construcción de un mundo justo y sostenible, que genere y participe de un debate público e informado sobre las cuestiones que afectan al desarrollo, y que promueva la organización y la movilización social y la incidencia política para actuar a favor de la justicia global. 


\title{
1.2. El potencial de la cooperación descentralizada municipalista para el ámbito no formal de la $E D$
}

La Estrategia sostiene que la cooperación descentralizada y en concreto la que realizan los gobiernos locales puede crear escenarios idóneos para la promoción de la ED tal y como se ha definido anteriormente. La importancia y potencial de esta cooperación se ha reconocido por organismos internacionales, a través de la Agenda 2030 de 2015; por organismos estatales, a través, por ejemplo, del v Plan Director de la Cooperación Española de 2018; y por organismos autonómicos, como muestra el IV Plan Director de la Comunitat Valenciana de 2017, que destaca el potencial de los municipios para acompañar y fortalecer procesos de desarrollo. La literatura nos habla además de los numerosos puntos fuertes de la cooperación descentralizada, de gran interés para el desarrollo de la ED no formal:

\begin{abstract}
Podríamos condensar como puntos fuertes su autonomía, su vinculación con el movimiento asociativo, el apoyo que es capaz de reunir, la apertura hacia múltiples actores, su capacidad de certeza, el respaldo social que es capaz de concitar, la cualificación de sus actuaciones, el apoyo al municipalismo, la solidaridad ciudadana que puede reunir. (Gómez, 2010, p. 7)
\end{abstract}

Sin embargo, la cooperación descentralizada también presenta desafíos y obstáculos que han de ser tenidos en cuenta si se quiere llevar a cabo procesos de ED de calidad. Por ejemplo, Gómez (2010) se refiere a riesgos como la fragmentación del discurso, las acciones puntuales y aisladas, la repetición de campañas o la incoherencia entre políticas, como algunos de los elementos negativos que pueden darse.

Esta Estrategia parte del reconocimiento de la importancia, así como de los puntos fuertes y los riesgos de la cooperación descentralizada municipalista, para desarrollar una ED generadora de ciudadanía activa. Asimismo, reconoce que las ciudades pueden ser espacios muy propicios para desarrollar iniciativas de ED en el ámbito no formal. Por ámbito no formal podemos entender, de acuerdo con Argibay y Celorio (2005):

Todas aquellas acciones con explícita intención formativa. Constituye un escenario de formación flexible en donde las prácticas educativas son más libres, participativas y dialógicas que en la educación reglada. (p. 63-64)

Si bien buena parte de los esfuerzos en materia de ED se han realizado tradicionalmente en el ámbito formal, es creciente el interés y el reconocimiento del enorme potencial de los procesos no formales e informales de aprendizaje para la construcción de ciudadanía.

Este interés fue el que animó al Programa de Cooperación al Desarrollo, perteneciente a la Concejalía de Cooperación al Desarrollo y Migración del Ayuntamiento de la ciudad de Valencia, a proponer la realización de la Estrategia involucrando no sólo a las ONGD, que tradicionalmente han sido los agentes principales de la ED (Mesa, 2000), sino también a los servicios y recursos municipales del Ayuntamiento. En concreto, el Programa de Cooperación lanzó una invitación específica a los Centros Municipales de Juventud, a las Bibliotecas Municipales y a los centros de la Universidad Popular para que, junto con las ONGD, se implicaran en el proceso de realización de la Estrategia. La sección siguiente describe esta implicación y el proceso participativo, que culminó con la aprobación de la Estrategia por el Pleno del Ayuntamiento de Valencia en septiembre de 2018.

\subsection{Metodología de realización de la estrategia}

Desde el mes de septiembre de 2017 a julio de 2018 , las autoras de este artículo lideraron y participaron activamente en el proceso de desarrollo de la Estrategia. Este se realizó en 
dos etapas: una primera (de septiembre de 2017 a enero de 2018) en la que se llevó a cabo un diagnóstico y una segunda (de febrero a julio de 2018) en la que se diseñó la Estrategia.

El objetivo del diagnóstico era cuádruple: por un lado, mapear la visión de la ED de distintos agentes de la ciudad de Valencia, potencialmente impulsores de acciones de ED en el ámbito no formal; por otro lado, se trataba de conocer los recursos de los que disponían, así como los procesos educativos en los que participaban; asimismo, se querían entender las condiciones de contex to que podrían facilitar u obstaculizar la realización de acciones de ED. Por último, se trataba de identificar ideas fuerza que pudieran ir perfilando las bases de la Estrategia.

El diagnóstico se articuló en 5 fases que se recogen en el cuadro 1. En todo momento y, en coherencia con la visión de una ED impulsora de una ciudadanía global, se prestó especial atención a que el proceso de elaboración del diagnóstico fuera realmente participativo y permitiera integrar efectivamente las opiniones y perspectivas de las personas con las que se iba interaccionando.

Cuadro 1. Fases del diagnóstico

\begin{tabular}{|c|c|c|c|c|c|}
\hline $\begin{array}{l}\text { FASES DEL DIAGNÓSTICO } \\
\end{array}$ & SEPTIEMBRE & OCTUBRE & NOVIEMBRE & DICIEMBRE & ENERO \\
\hline $\begin{array}{l}\text { Recopilación y análisis de } \\
\text { documentación secundaria }\end{array}$ & & & & & \\
\hline Generación de información primaria & & & & & \\
\hline Análisis de la información & & & & & \\
\hline Elaboración del diagnóstico & & & & & \\
\hline $\begin{array}{l}\text { Contraste y presentación del } \\
\text { diagnóstico }\end{array}$ & & & & & \\
\hline
\end{tabular}

Fuente: Elaboración propia a partir de Boni y otros (2018).

A lo largo de la realización del diagnóstico, se revisó y analizó documentación secundaria sobre ED a nivel global, europeo, estatal y valenciano, así como las políticas municipales en materia de ED de dos ciudades, Barcelona y Bilbao. Asimismo, se realizaron 23 entrevistas en profundidad (17 a servicios y recursos municipales y 6 a ONGD) y un taller específico sobre ED no formal en el espacio de “Diálogos” del encuentro Àgora organizado por el Ayuntamiento de Valencia, con una participación de 40 personas. Posteriormente, se realizó un taller específico para recabar reflexiones y propuestas para el diagnóstico, en el Centro Municipal de Juventud de Patraix, con una participación de 26 personas.
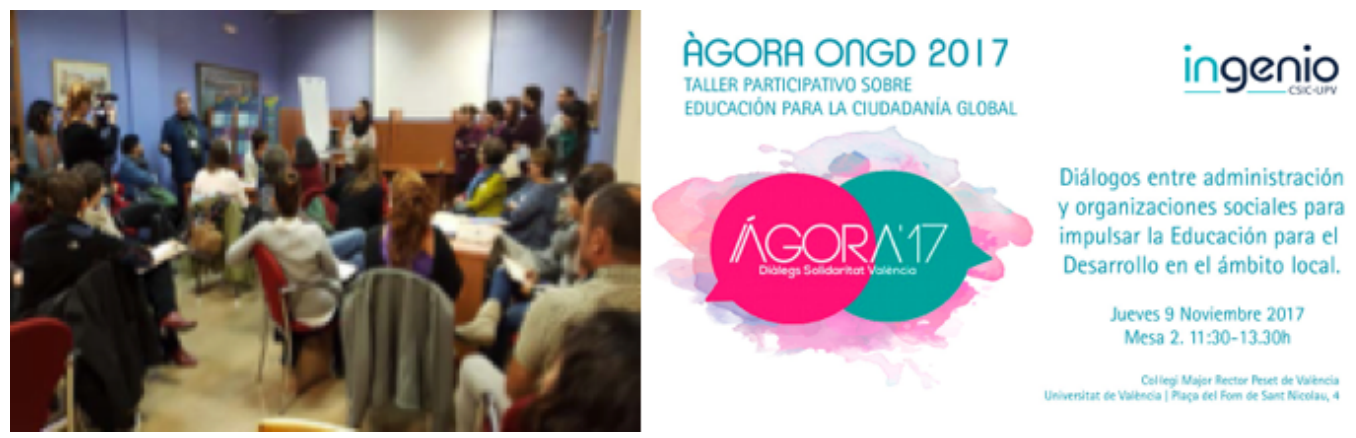

Figura 1. Taller multiactor en el espacio del Ágora 2017

Fuente: Recuperado de Boni y otros (2018). 
Tras ello, se realizó el análisis y se obtuvieron las ideas fuerza que posteriormente informaron la Estrategia (ver apartado siguiente). Tras ello, se realizó una presentación a la Comisión de ED del Consejo de Cooperación al Desarrollo municipal y, por último, se llevó a cabo la presentación pública del diagnóstico en la Biblioteca municipal de Abastos, para la cual se elaboró un documento de recomendación de políticas.

La estrategia se realizó en 4 fases, las cuales se recogen en la figura 2 , y tuvo el mismo carácter participativo que caracterizó el diagnóstico.

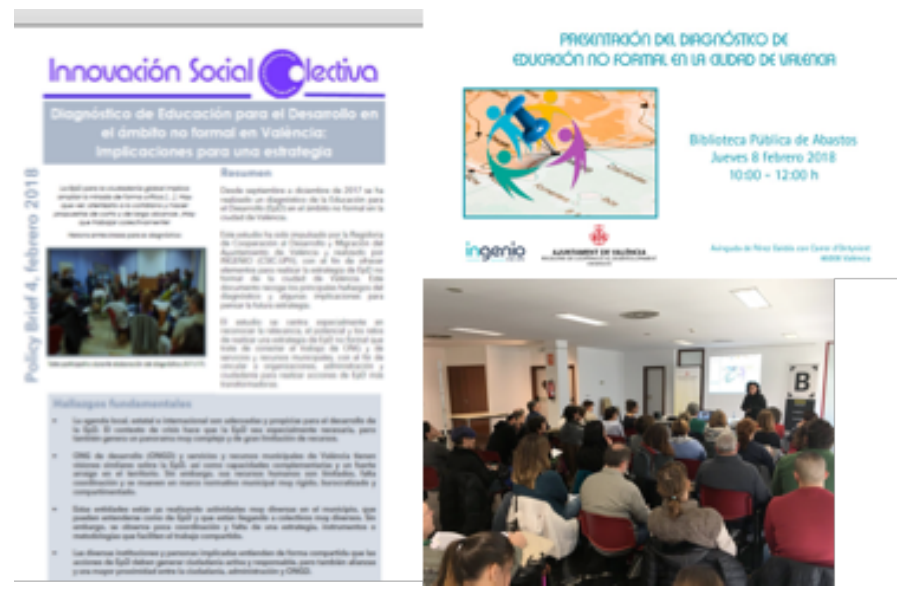

Figura 2. Policy brief y presentación del diagnóstico

Fuente: Recuperado de Boni y otros (2018).

Se comenzó con la realización de entrevistas en profundidad a personal de los servicios municipales, que sirvieron para contrastar lo obtenido en el diagnóstico y proponer unos lineamientos claves que se discutieron en un taller conjunto celebrado en la Universidad Popular de Rovella, en el que participaron 26 personas pertenecientes a ONGD y servicios del ayuntamiento (cuadro 2, figura 3).

Cuadro 2. Fases de la elaboración de la estrategia

\begin{tabular}{|c|c|c|c|c|c|c|}
\hline FASES DE LA ESTRATEGIA & FEBRERo & MARZO & ABRIL & MAYO & JUNIO & JULIO \\
\hline $\begin{array}{l}\text { Realización de entrevistas en profundic } \\
\text { Servicios y taller con ONGD }\end{array}$ & & & & & & \\
\hline Primeros lineamientos de la estrategia & & & & & & \\
\hline Taller conjunto & & & & & & \\
\hline $\begin{array}{l}\text { Escritura de la Estrategia, contraste y } \\
\text { presentación }\end{array}$ & & & & & & \\
\hline
\end{tabular}

Fuente: Elaboración propia a partir de Boni y otros (2018).
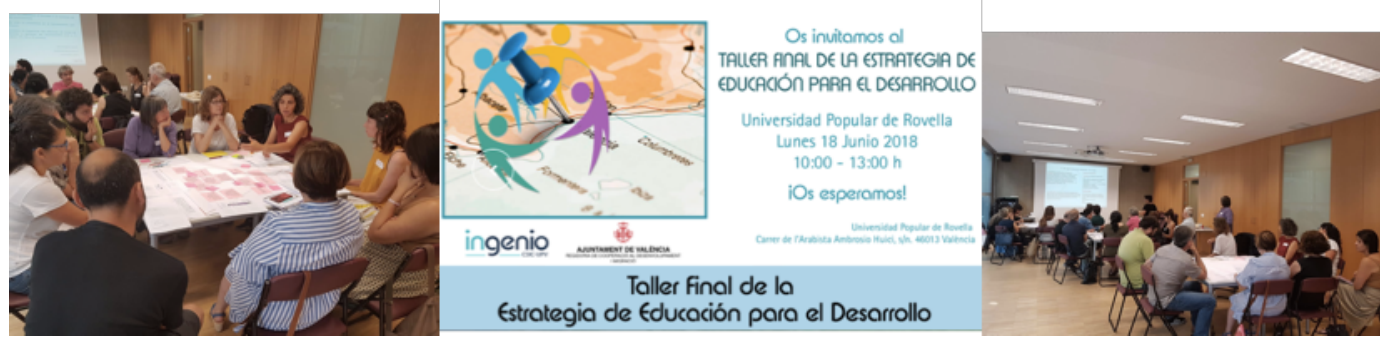

Figura 3. Taller multi-actor para la elaboración de la estrategia

Fuente: Recuperado de Boni y otros (2018). 
Posteriormente, se elaboró el documento de la Estrategia que se presentó ante el Consejo Local de Cooperación al Desarrollo del Ayuntamiento de Valencia en julio de 2018 y que fue posteriormente aprobada por el Pleno del Ayuntamiento el 27 de septiembre de 2018.

\subsection{4. Ámbitos de intervención principales de la estrategia}

El diagnóstico realizado permitió identificar seis ámbitos clave que fueron debatidos en los diferentes espacios de participación creados en el proceso y que, posteriormente, darían lugar a la definición de los ejes principales de la Estrategia. A continuación, se resumen las principales características de los seis ámbitos.

\section{Aprovechar la educación para la ciudadanía global como concepto cohesionador}

Los resultados del diagnóstico sugirieron que, para construir procesos de ED no formal en Valencia que conecten a distintos actores, puede resultar de gran interés aprovechar la propia idea de Educación para la Ciudadanía Global como término "paraguas" o aglutinante. Puede ser un referente común para que distintos actores realicen acciones en el territorio vinculadas a cuestiones de valores, solidaridad o justicia global. Aunque los y las profesionales municipales emplean términos diferentes al de Educación para la Ciudadanía Global, que es más propio de los actores que operan en el sistema de cooperación, existen visiones compartidas sobre la educación que se busca. Esta estaría orientada a generar conocimientos, valores y actitudes para una ciudadanía más conscientes, y a generar participación, acción y compromiso en la vida social y política. En este sentido, están emparentados los distintos conceptos que se emplean: ED, educación en valores, educación comunitaria, educación para la justicia global, educación para la transformación social... Lo relevante sería que el término tenga un sentido transformador compartido y que las agentes puedan sentirse identificadas con el mismo.

\section{Construir alianzas como medio y como fin}

Uno de los elementos más repetidos es la necesidad de formar alianzas entre las entidades públicas y privadas como un medio para poder afianzar procesos de Educación para la Ciudadanía Global en el ámbito no formal de la ciudad de Valencia. Además, se han señalado también frecuentemente las alianzas como un fin deseable de las acciones de Educación para la Ciudadanía Global. Se trataría, como han insistido varios actores durante el proceso del diagnóstico, de construir alianzas no sólo puntuales o instrumentales, sino estratégicas y de largo plazo.

Esto puede implicar, entre otras cuestiones, reconocer a los agentes que ya están trabajando en temáticas relacionadas con la Educación para la Ciudadanía Global; construir espacios de coordinación; compartir información y recursos; conocer y alinear objetivos y estrategias educativas. Por otro lado, el diagnóstico evidenció la voluntad de las distintas participantes por propiciar espacios de encuentro interdepartamentales, entre ONGD y servicios y entre servicios, ONGD y concejalías.

La interdisciplinariedad de la Educación para la Ciudadanía Global supone, además, una oportunidad y una ventaja para desarrollar las alianzas que no se dan en este ni en otros temas, e ir así en contra de las dinámicas e inercias habituales. Generar una ciudadanía global, que sea consciente de los problemas de la justicia social y de su papel activo en su entorno cercano, es un objetivo primordial que puede ser apropiado por muchos de los servicios municipales. 


\section{Aprovechar y fortalecer el arraigo en el territorio}

Generar procesos de Educación para la Ciudadanía Global en el ámbito no formal necesariamente supone tener en cuenta las particularidades, capacidades e intereses tanto de agentes estratégicos como del público objetivo de sus acciones. Estas particularidades se encuentran necesariamente vinculadas a las características socio-culturales del territorio donde se ubican. Se diría que una estrategia de Educación para la Ciudadanía Global debería hacer especial énfasis en aprovechar la presencia de los servicios y recursos en el territorio; su conocimiento de las comunidades y de sus problemas e intereses y las conexiones con el tejido asociativo local.

Esto puede permitir plantear acciones no solo más pertinentes, sino también más transformadoras, al vincular las acciones que desarrollen los procesos educativos con la comprensión de los problemas de los barrios y con la identificación de soluciones. Esto puede reforzar la visión "glocal" (global-local) y la dimensión movilizadora que la Educación para la Ciudadanía Global pretende a la hora promover procesos educativos transformadores.

Por otro lado, la propia diversidad en los barrios, siendo también un reto para plantear acciones, puede de hecho fortalecer y enriquecer los procesos de Educación para la Ciudadanía Global.

Reforzar el rol de los recursos y servicios como espacios de dinamización sociocultural

Otro elemento recurrentemente señalado ha sido la voluntad de los servicios y las ONGD por sentirse reconocidos en su labor como agentes activos dinamizadores y generadores de cambios sociales en la vida de los barrios. En este sentido, las acciones de Educación para la Ciudadanía Global en el ámbito no formal deberían aprovechar la motivación de servicios y organizaciones por avanzar en cambiar su rol, ayudar a fortalecerlo, y contribuir también a transformar la visión de la ciudadanía respecto a estos agentes y recursos.

\section{Generar diversidad e innovación en el tipo de acciones y las metodologías}

El desarrollo de una estrategia de Educación para la Ciudadanía Global se puede ver como una oportunidad para diversificar las acciones en varios sentidos: 1) diferenciar los y las agentes involucrados en la Educación para la Ciudadanía Global y reconocer algunos que quizá desarrollaban acciones educativas transformadoras con otra denominación y sin apoyo de organismos de cooperación; 2) ampliar el público al que se llega, ya que la conexión con el territorio puede permitir llegar a un rango generacional y a un perfil sociocultural más diverso y 3) diversificar el tipo de actividades que se llevan a cabo.

Por otro lado, el diagnóstico indicó que el despliegue de acciones de Educación para la Ciudadanía Global puede resultar mucho más transformador en tanto adopte metodologías activas y participativas que promuevan el debate, el diálogo y la relación con los problemas del entorno. Se trata, además, de metodologías con las que están familiarizados tanto las ONGD como profesionales de los varios recursos del ayuntamiento.

Asimismo, parece importante pensar una estrategia que impulse no solo acciones específicas, sino también la transversalidad de la Educación para la Ciudadanía Global en las distintas acciones de los recursos y servicios del ayuntamiento. Esto implica, tanto introducir temáticas y enfoques propios de la Educación para la Ciudadanía Global en otras acciones de educación no formal, como considerar como acciones de Educación para 
la Ciudadanía Global algunas actividades que ya existen e incorporan estos temas o enfoques.

Por último, cabe destacar que lo anterior implica pensar también en acciones y procesos educativos de largo plazo. Como señalan algunas personas entrevistadas, el aprendizaje transformador no se genera de forma inmediata, sino que necesita de un periodo largo de tiempo para interiorizar los nuevos saberes y los nuevos hábitos.

Construir sobre temas clave de los servicios y explorar abrir nuevos.

Si bien todas las esferas de la vida social pueden ser abordadas por la Educación para la Ciudadanía Global y convertirse en objeto de reflexión, parece oportuno desplegar una estrategia que se construya sobre temas en los que los varios actores implicados tienen interés, trayectoria y experiencia. Según lo identificado en el diagnóstico, aparecieron dos temas clave: en primer lugar, la cuestión del género. Con distintas aproximaciones y énfasis, se trata de una cuestión trabajada y conocida en todos los servicios y ONGD involucrados en el diagnóstico, ya sea como un tema en sí mismo o como una temática transversal. A este hecho se suma el esfuerzo de la Administración pública local y autonómica en los últimos años por introducir el enfoque de género en su funcionamiento. También, la creciente importancia del tema en el debate público en general. En segundo lugar, la cuestión de la diversidad cultural y la interculturalidad. También el diagnóstico reveló que estos temas son centrales para la actividad de distintos agentes, aunque en menor medida que la cuestión del género.

Por otro lado, el despliegue de acciones de Educación para la Ciudadanía Global no formal en las que colaboren servicios del ayuntamiento y ONGD podría explorar nuevas temáticas que no han trabajado algunos de los actores. Como ejemplo, cuestiones como la sostenibilidad, el comercio justo o la soberanía alimentaria, muy trabajadas en el ámbito de las ONGD valencianas, pero en mucha menor medida por los servicios municipales que han participado en el diagnóstico. Otros ejemplos tendrían que ver con cuestiones o enfoques que han trabajado algunos servicios o recursos, pero no de manera tan frecuente las ONGD, como es el caso del uso de tecnologías de la información y comunicación y juventud. En cualquier caso, la incorporación de nuevas temáticas puede a su vez ser un estímulo para renovar el tipo de cuestiones trabajadas por algunos servicios municipales.

\subsection{Ejes principales de la estrategia}

Los ámbitos de intervención clave de la Estrategia fueron cotejados y discutidos con los servicios municipales y las ONGD en los espacios y momentos indicados en la metodología, dando lugar a una propuesta de 3 ejes principales (la "tríada", según la denominamos) en cuyos vértices figuran los tres principios claves: Formación en temáticas y metodologías, Territorio y Coordinación

El primer eje se refiere a la formación de los diversos agentes, tanto en temáticas como en metodologías de la Educación para la Ciudadanía Global: formación dirigida a personal del Ayuntamiento de Valencia (tanto funcionario como no), personal de ONGD (tanto profesional como no) y otros actores tanto públicos como privados. Se ha destacado en numerosas ocasiones que el enfoque de la Educación para la Ciudadanía Global tiene que estar presente en todas las iniciativas. 
Las temáticas identificadas como prioritarias fueron las siguientes:

- Los Objetivos de Desarrollo Sostenible y su relación con las políticas públicas y las temáticas de la Educación para la Ciudadanía Global.

- Género.

- Los mecanismos de participación ciudadana existentes a nivel municipal y el propio concepto de participación ciudadana.

- Los derechos humanos.

- La soberanía alimentaria y las iniciativas ciudadanas orientadas a la producción y el consumo alimentario sostenible (huertos urbanos, mercados locales ecológicos).

- Las migraciones y refugio.

- La xenofobia y la homofobia.

- La educación para el consumo.

Como metodologías más adecuadas, sin ánimo excluyente de otras posibles, se destacaron:

- Aquellas que permitan identificar iniciativas en los barrios (mapeos, diagnósticos, etc.).

- Metodologías que favorezcan conectar iniciativas y personas y establecer relaciones en los diferentes territorios (por ejemplo: aprendizaje-servicio, cartografía social, video participativo, etc.)

- El trabajo con perspectivas y metodologías que incorporen el arte.

- Metodologías que permitan fortalecer la comunicación y divulgación de lo que se realiza como ED.

- Metodologías que contribuyan a profundizar en el trabajo en equipo, la motivación, los cuidados en el trabajo compartido, etc.

El segundo eje se refiere a incorporar una perspectiva territorial como forma de actuación. Cuando se habla de territorio, nos referimos no sólo a los elementos físicos del barrio o distrito, sino también a otros espacios (de relaciones, socio-culturales, etc.) que pueden materializarse. Se entiende que el territorio, además de una dimensión física y material, tiene además una dimensión simbólica, es decir, una serie de significados para las personas y colectivos que participan o pueden participar en los procesos. Por último, el territorio se entiende desde un enfoque global-local, que permite aproximarse al territorio de la ciudad de Valencia en conexión con otros territorios del Sur y Norte global.

Esta mirada pone en consecuencia el foco en las iniciativas, colectivos, instituciones que existen en los territorios y que serían las protagonistas en el impulso de las acciones de la Estrategia. Entre ellas, destacan agentes de la Administración Pública como pueden ser la Concejalía de Cooperación al Desarrollo y Migración, la Comisión de ED del Consejo Local de Cooperación al Desarrollo, los Centros Municipales de Juventud, las Bibliotecas, la Universidad Popular con sus diferentes centros, las Juntas de Distrito, los centros de mayores, los centros educativos, los servicios y secciones de otras Concejalías (por ej., aquellas con competencias en participación, educación, juventud o relación con los medios de comunicación, entre otras) y las Universidades. 
Por parte de la sociedad civil, algunos de los actores (organizados o no) con presencia territorial podrían ser las ONGD, los colectivos sociales, culturales y recreativos del barrio, monitoras y animadoras juveniles y otros agentes multiplicadores, las Mesas por la Solidaritat, periodistas, jóvenes, personas mayores, vecinos y vecinas en general.

El tercer eje nos remite a la necesidad de coordinación que se tendría que dar en diferentes ámbitos. Esta coordinación puede incluir distintos niveles y afectaría no solo a la relación entre agentes, sino a la conexión entre normativas de diferente nivel. La coordinación se debería dar entre las diferentes concejalías del Ayuntamiento, en los diferentes actores de los territorios, entre el Ayuntamiento y los actores de la sociedad civil que participan de la Estrategia y entre los actores públicos del espacio local y autonómico.

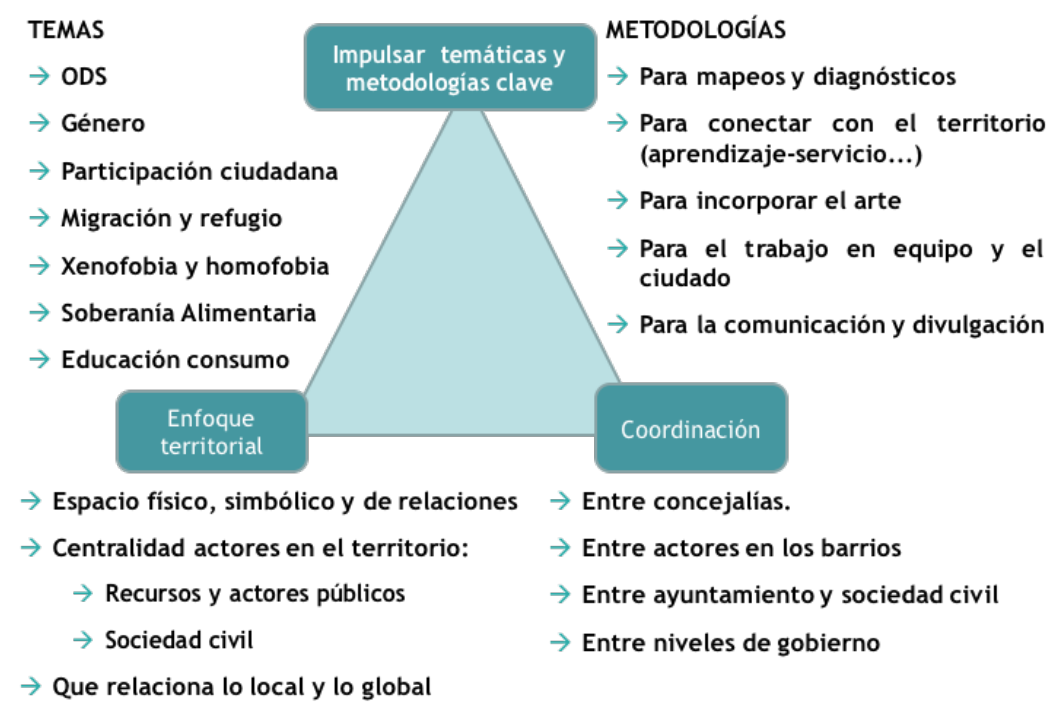

Figura 4. Ejes de la estrategia

Fuente: Recuperado de Boni y otros (2018).

Los tres ejes de la Estrategia han dado lugar a 10 líneas específicas de actuación que van desde la creación de espacios de coordinación, a la creación de nuevas convocatorias y la modificación de las anteriores. Excede de la contribución de este artículo la descripción de estas líneas de actuación, puesto que lo que nos interesa resaltar es cómo esta política pública puede contribuir a la realización de los ODS. Para ello nos apoyaremos en una interpretación diferente de los ODS, que se propone desde la perspectiva de la innovación transformativa. Este será el objeto de la siguiente sección, para posteriormente utilizar este marco para pensar en la contribución de la Estrategia a los ODS.

\subsection{Un marco diferente para interpretar los oDs desde la política de innovación transformadora}

Los 17 ODS establecidos en la Agenda 2030 de las Naciones Unidas desglosan 169 metas y 232 indicadores que resumen los principales desafíos de nuestro mundo. Su implementación requiere contribuciones importantes de la política de Ciencia, Tecnología e Innovación (CTI), como lo reconoce la ONU al incluir explícitamente la CTI en el ODS 9, identificándola como un mecanismo clave para la implementación de todos los ODS. 
Dentro del Consorcio de Política de Innovación Transformativa (TIPC, por sus siglas en inglés ${ }^{1}$, los miembros han aceptado este desafío, en un trabajo transnacional conjunto para proporcionar una nueva base transformadora para la política de CTI. Ellos reconocen que se requiere apertura y voluntad para explorar nuevos principios e ideas transformadoras. Se trata de proponer una forma de ver la implementación de los ODS desde una perspectiva de política de innovación transformadora, que es diferente y complementaria al proceso predominante de integración de los ODS en las políticas actuales. La innovación transformadora ofrece un enfoque integrado y de sistema orientado a las conexiones subyacentes y las compensaciones entre los ODS. En lugar de tratar los ODS como objetivos individuales o verlos como misiones, se centra en los procesos de transformación que dan lugar a los resultados específicos definidos por el conjunto de ODS.

Los ODS necesitan, no solo un enfoque sobre un nuevo tipo de innovación, la transformación de los sistemas socio-técnicos, sino también un replanteamiento de la política de CTI. La formulación e implementación de una política de CTI de este tipo puede ser una acción estratégica clave para el cumplimiento de la Agenda 2030. Si la transformación se entiende como la necesidad de cambiar fundamentalmente múltiples sistemas socio-técnicos, la innovación transformadora ofrece una nueva forma de ver los oDs y sus relaciones mutuas, una que es diferente del proceso generalmente aceptado de integración de los ODS en las políticas actuales.

Desde el punto de vista de una política de CTI transformadora se podrían distinguir tres tipos de ODS (ver figura 5):

- ODS que cubren una gama específica o más amplia de sistemas sociotécnicos o áreas de aplicación. Por ejemplo, ODS 3 sobre salud, ODS 4 sobre educación, ODS 6 sobre agua potable y saneamiento, ODS 7 sobre energía limpia y asequible; también ODS 9 sobre Innovación, industria e infraestructura, ODS 11 sobre ciudades y comunidades sostenibles, ODS 14 vida debajo del agua y ODS 15 vida en la tierra, todos ellos están directamente relacionados con múltiples sistemas.

- ODS que enfatizan "direcciones transversales" o direccionalidad. En este caso: ODS 1 fin de la pobreza; ODS 2 fin al hambre; ODS 5 igualdad de género; ODS 8 trabajo decente y crecimiento económico; ODS 10 desigualdades reducidas; ODS 12 producción y consumo responsables y ODS 13 acción climática.

- ODS que se centran en el cambio estructural de las condiciones marco necesarias para lograr la transformación. Esto incluye cambiar los acuerdos de gobernanza entre el Estado, el mercado, la sociedad civil y la ciencia. Estos se expresan en los dos ODS restantes: ODS 16 paz, justicia e instituciones fuertes y ODS 17 alianzas para los ODS.

Estas distinciones permitirían a países, regiones y organizaciones internacionales concentrar sus esfuerzos en la transformación de los sistemas socio-técnicos, utilizando los ODS no sólo para decidir sobre qué sistemas, sino también qué direccionalidades incluir

\footnotetext{
${ }^{1}$ La descripción de TIPC puede verse en www.tipconsortium.net Se trata de un consorcio internacional compuesto por 6 países (Suecia, Finlandia, Noruega, Colombia, Méjico y Sudáfrica) y liderado por la Science and Policy Research Unit de la Universidad de Sussex en Reino Unido. El objetivo de este consorcio es transformar las políticas de innovación de los distintos países para que puedan abordar los desafíos sociales y ambientales. Una de las autoras de este trabajo participa activamente en este Consorcio y ha sido co-autora de la propuesta que se describe en esta sección.
} 
en el proceso (figura 5). También llevaría a cuestionar si las condiciones marco, incluidas las capacidades transformadoras, existen para llevar a cabo dichas transformaciones. El punto de partida para el proceso no sería incorporar políticas existentes, sino crear espacios, expectativas, coaliciones y aprendizaje profundo para un cambio transformador.

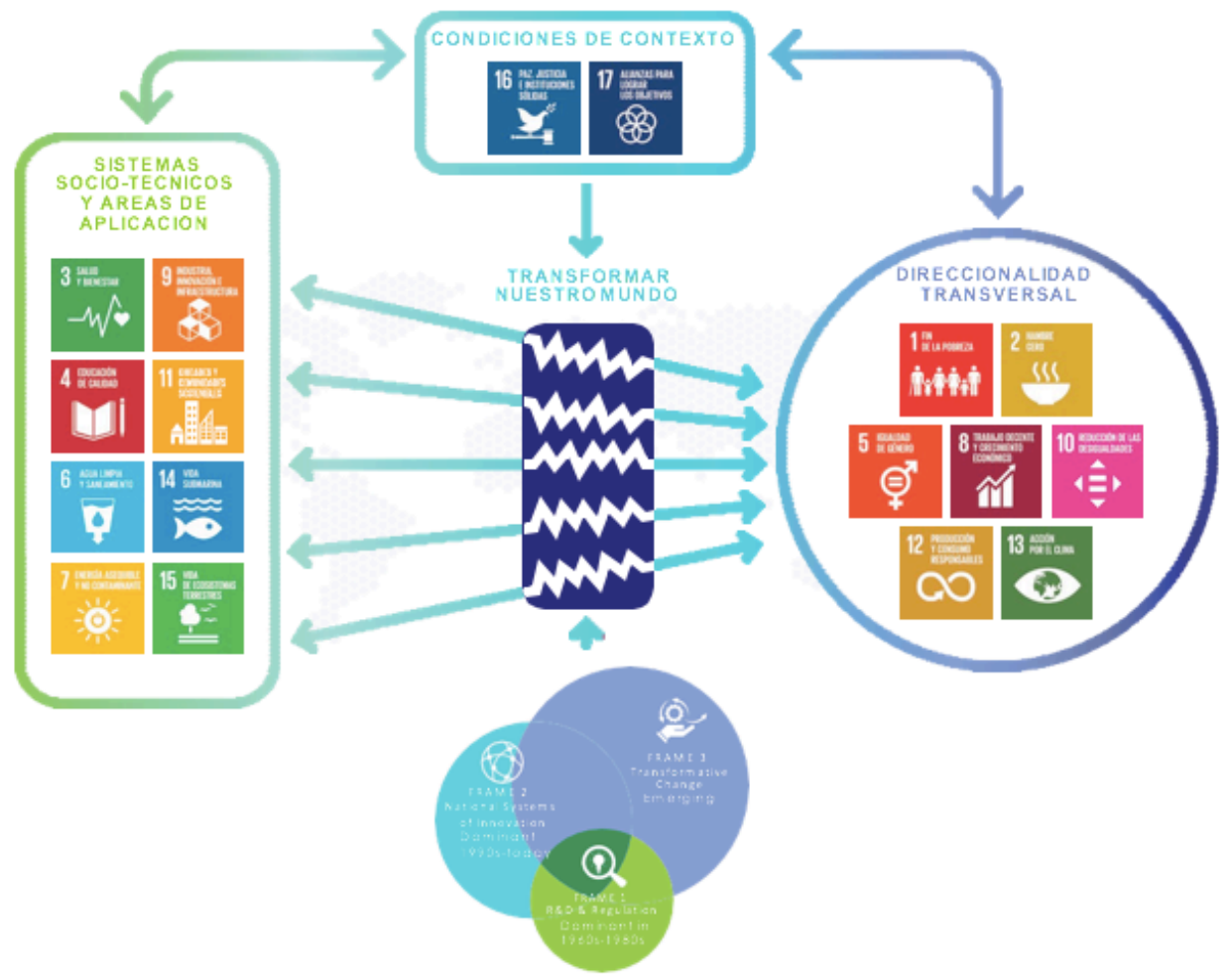

Figura 5. Abordando los objetivos de desarrollo sostenible a través de la política de innovación transformativa

Fuente: Recuperado de Shol y otros (2018).

Así, por ejemplo, si una política CTI pretendiera transformar el sistema socio-técnico de la energía (ODS 7), habría que plantearse la direccionalidad de esta transformación y ver si dicha transformación se dirige hacia una mejora de la equidad de género (ODS 5), o aborda la reducción de las desigualdades (ODS12) a la par que pretende afrontar los desafíos del cambio climático (ODS 13). Por otro lado, también se podría partir de los oDs que caracterizan la direccionalidad, y preguntarse cómo una política de CTI sobre energía, pensada para abordar los desafíos del cambio climático, puede incorporar otros oDs que definan su direccionalidad e incidir en la transformación de varios sistemas socio-técnicos. Asimismo, esta interpretación nos permite pensar en las condiciones marco de las transformaciones definidas por los ODS 16 y 17 y cómo una política de CTI puede considerarlas.

No es el objetivo de este trabajo entrar en más detalle en las contribuciones de las políticas de CTI a los ODS; nuestro ánimo es emplear este marco de análisis para pensar en las posibilidades que ofrece la Estrategia de ED de la ciudad de Valencia para abordar los ODS. Para ello, nos serviremos de los contenidos de los seis ámbitos de actuación de la 
Estrategia y los tres ejes clave que hemos caracterizado anteriormente. En la sección siguiente desarrollaremos en detalle nuestra interpretación.

\subsection{Adaptando los ODS a lo local mediante la ED}

La figura 6 representa las potenciales conexiones que podrían producirse entre los ODS y las transformaciones a las que puede contribuir la Estrategia de ED de la ciudad de Valencia definida por sus tres ejes clave.

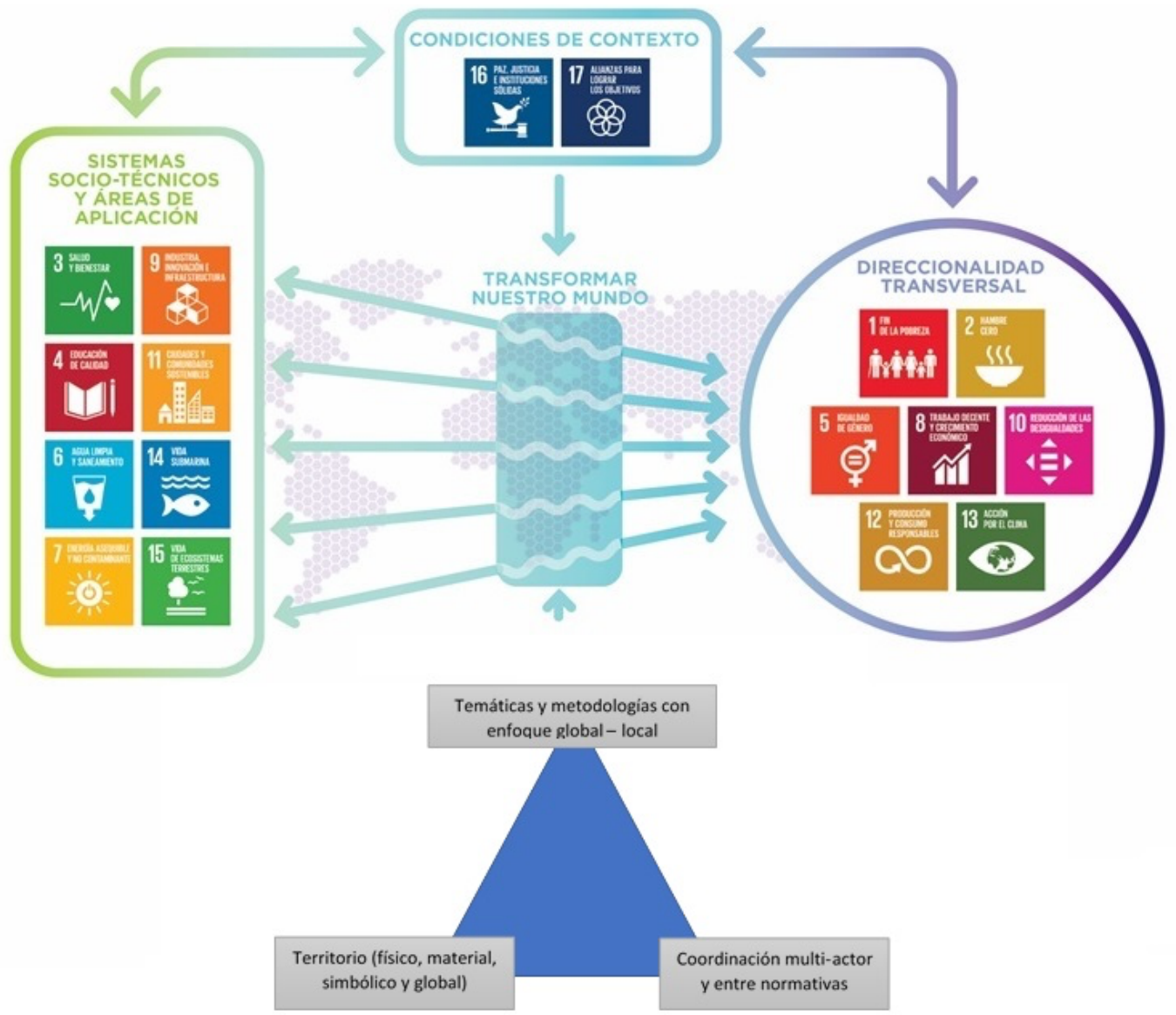

Figura 5. Objetivos de desarrollo sostenible y la estrategia de ED en el ámbito no formal de la ciudad de Valencia

Fuente: Elaboración propia.

En primer lugar, la propuesta de la Estrategia se trabajó en coordinación y con actores de la Administración municipal y de la sociedad civil. Esto contribuye a reforzar las condiciones de contexto necesarias para que se dé la transformación (ODS 16 y 17). En particular apunta al ODS 17, que es el relativo a las alianzas para lograr los ODS. Estas alianzas son fines y medios para la transformación. Por un lado, sin las necesarias redes y relaciones entre actores públicos y privados no es posible impulsar las acciones que plantea la Estrategia. Por otro lado, el tejer redes y relaciones puede ser a su vez un fin de las acciones transformadoras. Por ello, el oDs 17 constituye uno de los pilares fundamentales de la Estrategia.

En segundo lugar, el enfoque territorial nos da la pauta del nivel geográfico que necesita ser trasformado, en este caso los barrios de la ciudad de Valencia. Por ello, la Estrategia apuesta claramente por el ODS 11, que es el de las ciudades y comunidades sostenibles. 
Además de eso, al tratarse de procesos educativos transformadores, podríamos decir que el sistema socio-técnico por excelencia de la ED es el que recoge el oDs 4 que habla de educación. Es cierto que este ODS se dirige en mayor medida hacia la educación formal y que los actores protagonistas de la Estrategia no se encuentran fundamentalmente en este ámbito. Sin embargo, por un lado, los centros educativos pueden ser parte de las redes y alianzas que propone la Estrategia. Y, por otro lado, el hecho de que el ODS 4 se focalice sobre todo en la educación formal, no es más que el reflejo de una manera predominante de entender la contribución de la educación al desarrollo humano y sostenible, centrada básicamente en el acceso a la educación primaria y secundaria y a la formación técnica universitaria (Boni, Lopez-Fogues y Walker, 2016). La Estrategia se hace eco de otras propuestas educativas centradas en la educación comunitaria (Freire, 1993) que reivindican la importancia de los vínculos entre el proceso educativo y lo que ocurre en la comunidad. Es, asimismo, plenamente coherente con la propuesta de la CONGDE que describíamos en la sección primera de este artículo y que habla de la ED como un "Proceso de aprendizaje social compartido y como espacio de confluencia de actores orientados a la transformación social”. (CONGDE, 2011, p. 4).

En tercer lugar, el tipo de temáticas y metodologías que se proponen en la Estrategia también pueden contribuir a la transformación de otros sistemas socio-técnicos desde una determinada direccionalidad. Por ejemplo, el trabajar un proceso educativo con un eje soberanía alimentaria-género permitiría abordar el ODS 3 (salud), el ODS 5 (igualdad de género), el ODS 2 (Hambre cero), el ODS 12 (Producción y consumo responsables) además de los ya mencionados: ODS 11 (ciudades y comunidades sostenibles), ODS 4 (Educación) y ODS 17 (Alianzas). Otro ejemplo de temática educativa podría ser migración y refugio desde la participación ciudadana. Esto podría contribuir al oDs 13 (Reducción de las desigualdades) y a los ya mencionados ODS 11, ODS 4 y ODS 17 .

Los dos ejemplos anteriores muestran que hay temáticas más sinérgicas que otras. El hecho de tener esta perspectiva de interpretación de los ODS podría contribuir a una mayor transformación de los procesos educativos. Por ejemplo, añadiendo a la temática de refugio-migración y participación ciudadana, la perspectiva de género y un enfoque de derechos centrado en derecho del trabajo (ODS 8) o de salud (ODS 3).

\section{Conclusiones}

A lo largo de este texto hemos presentado una propuesta de ED que se recoge en la Estrategia para la Educación no formal de la ciudad de Valencia 2019-2022. El contenido de la Estrategia recoge una política pública municipal basada en tres ejes fundamentales: 1) un enfoque territorial en la unidad barrio o distrito de la ciudad de Valencia; 2) la importancia de la coordinación entre actores municipales que están en los barrios y las ONGD para desarrollar las propuestas educativas y 3) una serie de temáticas y metodologías prioritarias.

La relación de la Estrategia con los ODS ha sido interpretada empleando un marco de análisis pensado para la innovación transformadora desarrollado en el marco de un consorcio transnacional (Schot el al., 2018). Esta interpretación distingue entre oDS que definen los sistemas socio-técnicos o áreas de aplicación, los que definen la direccionalidad y los que indican las condiciones de contexto. 
Este marco ha permitido visibilizar las contribuciones que las acciones de ED que se definen en la Estrategia pueden realizar a los ODS. Existen tres ODS centrales en la propuesta de la Estrategia que son: ODS 11 que habla de las ciudades y comunidades sostenibles (eje territorial, ODS 17 que se refiere a las alianzas entre diferentes actores (eje coordinación) y oDS 4 que abarca el sistema educativo (eje sobre temáticas y metodologías educativas).

Por otro lado, diferentes oDS pueden ser afectados en función de las temáticas y metodologías escogidas. Con esta interpretación no se está sugiriendo que las propuestas de ED que emanen de la Estrategia deben abordar cuantos más ODS mejor. El valor añadido de esta interpretación es que puede contribuir a aumentar la reflexión sobre los objetivos de transformación de las acciones educativas huyendo de perspectivas compartimentadas que tanto han predominado en la interpretación de los ODS. Por otro lado, pone de manifiesto la importancia de las redes y coordinación entre actores como un elemento clave de la ED y la ciudad y sus barrios como los espacios que necesitan ser transformados y a partir de los cuales pueden partir las transformaciones.

No hemos entrado en la cuestión de cómo poder capturar si se están produciendo o no las transformaciones en los distintos ODS. Esta cuestión excede del objetivo de este artículo y requeriría de una profunda reflexión y análisis. Sin embargo, sugerimos que los indicadores que se proponen para la medición de los ODS no son adecuados para dar la medida de los cambios que la Estrategia propone. El hecho de adaptar la Agenda 2030 al ámbito municipal requiere plantearse que las transformaciones a nivel municipal son diferentes a las de ámbito nacional y que exceden del campo de la cooperación internacional, y más si se plantean desde una propuesta educativa como la de la Educación para la Ciudadanía Global.

\section{Agradecimientos}

Queremos agradecer al Programa de Cooperación al Desarrollo, perteneciente a la Concejalía de Cooperación al Desarrollo y Migración, del Ayuntamiento de València, la confianza y el apoyo económico al equipo que firma este artículo para el desarrollo de la Estrategia. Asimismo, queremos agradecer a los y las profesionales de los diversos servicios del Ayuntamiento y a las ONGD que se implicaron y contribuyeron significativamente en todo el proceso de realización de la Estrategia.

\section{Referencias}

Argibay, M. y Celorio, G. (2005). Educación para el desarrollo. Recuperdo de http://www.dhl.hegoa.ehu.es/ficheros/o000/0023/La_educacion_para_el_desarrollo.pdf

Asamblea General de NNUU. (2015). Transformar nuestro mundo: La agenda 2030 para el desarrollo sostenible. Recuperdo de http://www.un.org/es/comun/docs/?symbol=A/RES/70/1

Boni, A. (2011). Educación para la ciudadanía global. Significados y espacios para un cosmopolitismo transformador. Revista Española de Educación Comparada, 17, 65-85.

Boni, A. y León, R. (2013). Educación para una ciudadanía global: Una estrategia imprescindible para la justicia social. En Intermon Oxfam (Ed.), La realidad de la ayuda 2012 (pp. 214-239). Barcelona: Oxfam. 
Boni, A., Lopez-Fogues, A. y Walker, M. (2016). Higher education and the post-2015 agenda: A contribution from the human development approach. Journal of Global Ethics, 12(1), 17-28.

Boni, A., Belda, S., Calabuig, C., Millán, M. A. y Talón, A. (2018). Estrategia de educación para el desarrollo en el ámbito no formal de la ciudad de Valencia.Valencia: Ingenio.

Boni, A, Arias, B., López-Fogués, A., Calabuig, C., Belda, S. y C. Monge (2016). Estrategia de educación para el desarrollo en el ámbito formal de la Comunitat Valenciana. Recuperado de http://www.transparencia.gva.es/web/cooperacion/estrategia-de-epd

CONGDE. (2011). La educación para el desarrollo en las coordinadoras autonómicas de ONGD. Recuperado de http://coordinadoraongd.org/publicaciones/la-educacion-para-eldesarrollo-en-las-coordinadoras-autonomicas-de-ongd/

CONGDE. (2014). Posicionamiento sobre la importancia de la educación para el desarrollo y el rol de las ONGD en la construcción de la ciudadanía global. Recuperado de http://aragonparticipa.aragon.es/sites/default/files/taller_2_epd_ciudadania_global_201 4.pdf

Freire, P. (1993). Pedagogía de la esperanza. Ciudad de México: Siglo XXI Editores.

Gómez, C. (2010). Una visión panorámica de la cooperación descentralizada de las entidades locales. Vitoria-Gasteiz: Ayuntamiento de Vitoria-Gasteiz.

HEGOA. (2014). Cambiar la educación para cambiar el mundo. Recuperado de http://publicaciones.hegoa.ehu.es/publications/330

INCYDE. (2016). Diagnóstico para la estrategia de educación para la transformación social. Bilbao: Agencia Vasca de Cooperación para el Desarrollo.

Mesa, M. (2000). La educación para el desarrollo: Entre la caridad y la ciudadanía global. Papeles de Cuestiones Internacionales, 70, 11-26.

Ortega-Carpio, M. L., Cordón, R. y Sianes, A. (2013). Educar para la ciudadanía global en el espacio universitario. Buenas prácticas de colaboración entre ONGD y universidad. Madrid: Fundación ETEA.

Schot, J., Boni, A., Ramirez, M. y Steward, E. (2018). Addressing SDGs through transformative innovation policy. TIPC research briefing. Recuperado de http://www.tipconsortium.net/wpcontent/uploads/2018/06/4198_TIPC_research_brief_web-FINAL.pdf

\section{Breve CV de los autores}

\section{Alejandra Boni Aristizábal}

Doctora en Derecho por la Universidad de Valencia y Catedrática de la U. Politècnica de València. Es Subdirectora del Instituto INGENIO (CSIC-UPV), docente e investigadora experta en EpD, habiendo sido investigadora principal de quince proyectos y contratos de investigación nacionales e internacionales sobre educación y desarrollo. Cuenta con más de cincuenta publicaciones sobre temáticas de educación, desarrollo y cooperación internacional. Ha sido la coordinadora de la realización de la Estrategia de Educación para el Desarrollo en el ámbito formal para la Generalitat Valenciana y de la Estrategia del Ayuntamiento de Valencia. Es miembro del Consejo Valenciano de Cooperación. ORCID ID: https://orcid.org/oooo-0003-3945-4336. Email: aboni@ingenio.upv.es 


\section{Sergio Belda-Miquel}

Doctor en el Programa en Desarrollo Local y Cooperación Internacional de la UPV y profesor asociado del Departamento de Trabajo Social de la Universitat de València. Es investigador del Instituto Ingenio. Experto en organizaciones no gubernamentales, cooperación al desarrollo, innovación social y cambio climático. Investigador invitado en el Institute of Development Studies y en la Universidad de Oslo. Ha sido miembro del equipo que ha elaborado la Estrategia de Educación para el Desarrollo en el ámbito formal para la Generalitat Valenciana y la Estrategia del Ayuntamiento. ORCID ID: https://orcid.org/0000-0003-0164-5487. Email: serbelmi@ingenio.upv.es

\section{Carola Calabuig Tormo}

Doctora Ingeniera Industrial por la U. Politècnica de València y Profesora Titular de la misma. Directora Académica del Máster en Cooperación al Desarrollo por la UPV y experta en cooperación y sostenibilidad. Investigadora del Insituto Ingenio (CSIC-UPV). Ha sido Jefa de Planificación en el Ministerio de Asuntos Exteriores y Cooperación y miembro del equipo que ha elaborado la Estrategia de Educación para el Desarrollo en el ámbito formal para la Generalitat Valenciana y la Estrategia del Ayuntamiento. Email: cacator@dpi.upv.es

\section{María Alejandra Millán Franco}

Licenciada en Administración de Empresas en la Universidad Nacional de Colombia. Actualmente cursando el Máster en Cooperación al Desarrollo de la UPV. Ha sido miembro del equipo que ha elaborado el Diagnóstico de Educación para el Desarrollo en el ámbito no formal para el Ayuntamiento de València.Email: mamillanf@unal.edu.co

\section{Alba Talón Villacañas}

Licenciada en Sociología y Ciencias Políticas y de la Administración por la Universitat de València. Actualmente doctoranda en el Programa de Doctorado en Desarrollo Local y Cooperación Internacional por la Universitat Politècnica de València. Ha sido miembro del equipo que ha elaborado el Diagnóstico de Educación para el Desarrollo en el ámbito no formal para el Ayuntamiento de València. ORCID ID: https://orcid.org/0000-00016081-3415. Email: altavialba@gmail.com 